\title{
Individual pig object detection algorithm based on Gaussian mixture model
}

\author{
Li Yiyang, Sun Longqing*, Zou Yuanbing, Li Yue \\ (College of Information and Electrical Engineering, China Agricultural University, Beijing 100083, China)
}

\begin{abstract}
The background models are crucially important for the object extraction for moving objects detection in a video. The Gaussian mixture model (GMM) is one of popular methods in the background models. Gaussian mixture model which applied to the pig target detection has some shortcomings such as low efficiency of algorithm, misjudgment points and ghosts. This study proposed an improved algorithm based on adaptive Gaussian mixture model, to overcome the deficiencies of the traditional Gaussian mixture model in pig object detection. Based on Gaussian mixture background model, this paper introduced two new parameters of video frames $\mathrm{m}$ and $T_{0}$. The Gaussian distribution was scanned once every $\mathrm{m}$ frames, the excessive Gaussian distribution was deleted to improve the convergence speed of the model. Meanwhile, using different learning rates to suppress ghosts, a higher decreasing learning rate was adopted to accelerate the background modeling before $T_{0}$, the background model would become stable as the time continued and a smaller learning rate could be used. In order to maintain a stable background and reduce noise interference, a fixed learning rate after $T_{0}$ was used. Results of experiments indicated that this algorithm could quickly build the initial background model, detect the moving target pigs, and extract the complete contours of the target pigs'. The algorithm is characterized by good robustness and adaptability.
\end{abstract}

Keywords: object detection, individual pig, Gaussian mixture mode, background model, contours, behavioral trait DOI: $10.25165 /$ j.ijabe.20171005.3136

Citation: Li Y Y, Sun L Q, Zou Y B, Li Y. Individual pig object detection algorithm based on Gaussian mixture model. Int J Agric \& Biol Eng, 2017; 10(5): 186-193.

\section{Introduction}

The key elements of the natural pig behavior traits include feed and water intake frequency, and excretion frequency. These factors indicate the growth rate of species. The monitoring of behavioral traits and analyzing $^{[1]}$ are indispensable to determine the animal health. Pig detection is to segment the pig target from the background image, for the pig target classification, recognition and tracking, which is the basis of analysis of

Received date: 2016-12-16 Accepted date: 2017-03-20

Biographies: Li Yiyang, Master student, research interests: video image processing technology, Email: adelally@sina.com; Zou Yuanbing, Master student, research interests: video image processing technology, Email: zyb@cunbang.com; Li Yue, Master student, research interests: video image processing technology, Email: liyuelucky1@126.com.

*Corresponding author: Sun Longqing, Professor, research interest: computer technology. China Agricultural University, Beijing 100083, China. Email: sunlq@cau.edu.cn. the behavior characteristics of record.

Optical flow method, frame difference method and background subtraction method are the most commonly used methods for target detection ${ }^{[2-6]}$. The optical flow method $^{[7-11]}$ can detect object movement independently, but due to extensive data calculations, it becomes difficult for real-time monitoring. The frame difference method ${ }^{[12-14]}$ is simple but cannot track all the objects precisely. The background subtraction ${ }^{[15-19]}$ method based on the Gaussian model has the noise resistance and relatively accurate. Many researchers have improved the mixture Gaussian model algorithm. Bouttefroy et al. ${ }^{[20]}$ proposed a method to use different learning rate in the variance and mean of mixed Gaussian background model, setting specific variance to eliminate or reduce noise caused by illumination mutation and motion mutation. Kayabol and Kutluk ${ }^{[21]}$ proposed spatial joint spectral changes of Gaussian mixture model to solve hyper spectral image classification. The model provides 
a robust estimation for small sample training sets and determines the prior distribution of mean vector and covariance matrix to solve the parameter estimation problem. Moreover, it uses pixel space variation mix ratio to improve the classification accuracy rate. Shah et al. ${ }^{[22]}$ introduced a new local parameter learning algorithm for the Gaussian mixture mode background model, and used SURF feature matching algorithm to suppress the ghosts. Jie et al. ${ }^{[23]}$ presented a technique based on PCA and the Gaussian mixture model to segment color image of diseased wheat. This method sorted the image blocks according to the color feature, used Gaussian mixture model to calculate the new pixel sets formed by picked image blocks according to the sequence, and classified each pixel to the obtained Gaussian model to achieve the image segmentation of diseased leaves of wheat. Liu et al. ${ }^{[24]}$ put forward a dynamic background modeling method that using penalty factors in the Gaussian mixture model, which used the local update strategy to reduce the model complexity and to solve the problem of foreground ablation, and used the binary classification algorithm based on color deviation and brightness deviation to avoid the influence of object shadow area. However, Gaussian mixture mode ${ }^{[25]}$ has the following drawbacks: (1) The number of Gaussian distributions corresponding to each pixel in each frame remains fixed. In the real Gaussian background modeling scene, the changes of state corresponding to each scene in different area are different. As the scene changes, the number of states of the same area will change. If every pixel has the same number of models, the low efficiency of the algorithm can cause a lot of waste of resource, and it is difficult to achieve real-time monitoring. (2) The fixed learning rate is adopted in the model. When the learning rate and the scene speed do not match, the detection result will appear misjudgment points and ghosts. Stauffer and Grimson ${ }^{[26]}$ established an adaptive mixture Gaussian background model to update the model based on the online estimation. This could effectively solve the noise caused by external interference such as illumination changes and disordered background. Chen and Ellis ${ }^{[27]}$ presented an algorithm based on a self-adaptive Gaussian mixture to model the background of a scene imaged by a static video camera, the model used a dynamic learning rate with adaptation to global illumination to cope with sudden variations of scene illumination. Ren et al. ${ }^{[28]}$ introduced an adaptive learning algorithm for moving target detection based on Gaussian mixture model, the model of learning process was divided into two stages of initial formation and background maintenance updates, and adopted different learning strategies in different stage. Compared with other non-adaptive methods ${ }^{[29]}$, the Gaussian mixture mode does not need manual intervention in the initialization process, the background calculation error accumulation is small, and the background changes have a good adaptability. In order to increase the modeling convergence speed, this paper presents an improved algorithm using adaptive method to adjust the number of Gaussian distribution models, and using the adaptive learning rate to eliminate or reduce misjudgment points and ghosts.

\section{Gaussian mixture model}

The principle of Gaussian mixture model (GMM) states that due to the influence of environmental factors, the gray value of each pixel in the video image will change with the environment. It is generally observed that the background pixel gray values are consistent to the Gaussian distribution. This can be simulated by weighted sums of multiple Gaussian distributions. The Gaussian mixture model can be used to describe the change in the background of the video image. The change of the foreground object is random and generally does not obey the Gaussian distribution ${ }^{[26]}$. Based on this idea, if the pixel point satisfies the obtained Gaussian distribution model, it could be determined as the background, and vice versa.

Use $\eta\left(X_{t}, \mu, \Sigma\right)$ to represent the probability density function of Gaussian distribution, in which $\mu$ is the mean, $X_{t}$ is the current pixel value at time $t, \Sigma$ is the covariance matrix. The video sequence of particular pixel can be viewed as a time series. $\left\{X_{1}, X_{2} \ldots, X_{t}\right\}=\left\{I\left(x_{0}, y_{0}, i\right): 1 \leq i \leq t\right\}$ is used to describe the number of Gaussian distributions of each color point, respectively, as shown in Equation 


$$
\begin{aligned}
\eta\left(X_{t}, \mu_{i, t}, \Sigma_{i, t}\right)= & \frac{1}{(2 \pi)^{\frac{n}{2}}\left|\Sigma_{i, t}\right|^{\frac{1}{2}}} e^{-\frac{1}{2}\left(X_{t}-\mu_{i, t}\right)^{T} \Sigma_{i, k}^{-1}\left(X_{t}-\mu_{i, t}\right)} \\
& i=1,2, \cdots, K
\end{aligned}
$$

where, the subscript $t$ represents the time; $K$ is the number of Gaussian distributions functions; $\mu_{i, t}$ is the mathematical expectation (mean) of the $i^{\text {th }}$ Gaussian model; $n$ is the number of dimensions (usually referred to as the gray level information of the pixel) of the observed pixel vector $X_{t}$. To simplify the computation, it is assumed that the covariance matrix as Equation (2):

$$
\Sigma_{K, t}=\sigma_{K}^{2} I
$$

where, $I$ is the matrix unit; $\sigma_{K}$ is the standard deviation of the GMM distribution.

The probability of the current pixel value $X_{t}$ can be calculated using Equation (3):

$$
P\left(X_{t}\right)=\Sigma_{i=1}^{K} \omega_{i, t} \times \eta\left(X_{t} \mid \mu_{i, t}, \sigma_{i, t}\right)
$$

where, $\omega_{i, t}$ is the weight of the model $i$ in the Gaussian mixture model at time $t$.

New pixel $X_{t}$ in a video frame is matched with the existing Gaussian model distribution. If the gray value of the current pixel is close to the mean of a distribution, the pixel is considered to match the distribution, satisfying Equation (4):

$$
\left|X_{t}-\mu_{i, t-1}\right|<T H \sigma_{i, t-1}
$$

where, $T H$ is the threshold, generally set as 2.5 ; if the Gaussian distribution meet above conditions, the parameters are updated as Equations (5)-(8):

$$
\begin{gathered}
\omega_{i, t}=\left(1-\alpha_{i}\right) \omega_{i, t-1}+\alpha_{i} M_{i, t-1} \\
\mu_{i, t}=\left(1-\beta_{i}\right) \mu_{i, t-1}+\beta_{i} X_{t} \\
\sigma_{i, t}^{2}=\left(1-\beta_{i}\right) \sigma_{i, t-1}^{2}+\beta_{i}\left(X_{t}-\mu_{i, t}\right)^{T}\left(X_{t}-\mu_{i, t}\right) \\
\beta=\alpha \cdot \eta\left(X_{t}, \mu_{i, t}, \sigma_{i, t}\right)
\end{gathered}
$$

where, $\alpha$ is the model learning rate, generally determined by the experiment, $0 \leq \alpha \leq 1 ; \beta$ is the parameter learning rate; $M_{i, t-1}$ is a binary value equal to 1 if the current pixel $X_{t}$ matched a distribution and 0 otherwise. If the current pixel does not match a similar Gaussian distribution, a new Gaussian distribution is added to the background model to replace the Gaussian distribution with the lowest priority. The new Gaussian distribution will use a larger variance and a smaller initialized weight.
$K$ Gaussian distributions of each pixel are ranked from the highest to the lowest by the ratio of $\rho=\omega_{i} / \sigma_{k}$, and the models which are most likely to be the background of model is ranked at the forefront. The distributions caused by the unstable perturbation are at the bottom of the sequence, and will be eventually replaced by new Gaussian distributions. Select the former $B$ Gaussian distributions from $K$ Gaussian distributions as the background model using Equation (9):

$$
B=\arg \min n\left(\sum_{k=1}^{n} \omega_{i, K}>T\right)
$$

where, $T$ is the background threshold, generally set $T=$ 0.7; the best distribution to describe the background will be selected by setting the value of $T$.

\section{Improved algorithm}

\subsection{Improved Gaussian mixture model}

To meet the requirements of individual pig detection in this research by improving the Gaussian mixture background model. With the change of time, the weights of the Gaussian distributions which match in the Gaussian mixture model become higher. After the normalization, the weights of the Gaussian distributions which do not match become less, and the distributions with higher weights-variance ratios are used to describe the background distribution, the distributions with lower ratio describes the foreground distribution. When the weight $\omega_{j, t}^{i}$ of a Gaussian distribution is less than the initial value of $\omega_{0}$, and the $\omega_{j, t}^{i} / \sigma_{j, t}^{i}$ of the Gaussian component is smaller than the initial value of $\omega_{0} / \sigma_{0}$, the Gaussian distribution will be ranked after the new initialized Gaussian distribution. Continuing to hold this Gaussian distribution will make it possible to spend longer time learning the scene because when the scene corresponding to this Gaussian distribution shows up, this Gaussian distribution will be used to learn instead of a new Gaussian component. Redundant Gaussian components like this will affect the learning convergence rate of the model, and consume system computational resources. Through deleting the redundant Gaussian distributions after the updating and learning process of background model which each video frame handles this operation, the selection of the numbers of new adaptive 
Gaussian distributions will be improved. Considering the actual computational efficiency, this research adopted scanning Gaussian distribution every $m$ frames to detect the weights of every Gaussian distributions. If a Gaussian distribution satisfies Equation (10), the Gaussian distribution is determined to be a redundant Gaussian distribution, and deleted it.

$$
\left(w_{j, t}^{i}<w_{0}\right) \& \&\left(w_{0} / \sigma_{0}\right)<\left(w_{j, t}^{i} / \sigma_{j, t}^{i}\right)
$$

In the Gaussian mixture model, if the learning rate $\alpha$ is too small, the background update speed will slow down, and the phenomenon of a large number of false positives will appear in the test results; if $\alpha$ is too large, the model updates too fast, the target will easily integrate into the background, resulting in misjudgment points ${ }^{[28]}$. In order to accelerate the elimination of misjudgment points and ghosts, this paper proposes an adaptive learning rate strategy: with setting a threshold value of the frame number $T_{0}$, different learning rates are adopted in updating for the frames before $T_{0}$ and it is kept non-variant after frames $T_{0}$, as shown in Equation (11):

$$
\alpha=\left\{\begin{array}{l}
\frac{1}{2 t}, t \leq T_{0} \\
\frac{1}{2 T_{0}}, t>T_{0}
\end{array}\right.
$$

A higher learning rate might be used in the initial model to accelerate the updating speed to eliminate or reduce the ghosts; the background model will become stable as the time goes and a smaller learning rate will be used.

The updating mechanism of Gaussian mixture model can better deal with the slow background changes, but it is sensitive to the sudden changes such as illumination mutations. The sudden illumination mutations are shown as the changes of overall gray value, that is, the changes of difference between the current frame and the obtained average background gray value. When the difference is greater than a certain threshold, the parameters in the Gaussian distribution with the largest weight in all pixels of the whole image are replaced. $\mu_{0}$ (the mean of the GMM distribution) will be replaced by the image pixel value of current frame, $\omega_{0}$ (the weight of the GMM distribution)is set a larger value to ensure that the new distribution becomes stable background distribution, considering the indoor pigs as the research objects, the light does not change drastically. In this study, $\sigma_{0}$ (the standard deviation of the GMM distribution) was set 20 .

\subsection{Algorithm description}

The actual steps based on the improved Gaussian mixture model algorithm are as follows:

Step 1: Calculate the current learning rate, and establish a Gaussian mixture model for the initial video frame.

Step 2: Determine whether the new background matches the existing model. If it matches, then update the weights, mean and standard deviation values. If it does not match then add a new background model.

Step 3: Scan the Gauss distribution every 100 frames to detect weights of all Gaussian distributions. If a Gaussian distribution satisfies Equation (10) then determine that this Gaussian distribution is the redundant Gaussian distribution, and discard it.

Step 4: Normalize the weights of every Gaussian distribution and calculate the priority of Gaussian distribution and sort in descending order.

Step 5: Determine whether the current pixel matches the existing Gaussian distribution. If matches, then determine this pixel belongs to the background and foreground otherwise.

\section{Results and discussion}

\subsection{Experimental results and algorithm efficiency analysis}

In order to verify the effectiveness of the algorithm, this research used the VS2010 platform (opencv2.3.1) in Windows7 operating system, and compared the Gaussian mixture model and the new approach (Figure 1). The experimental results showed that the improved algorithm was more real-time. The processing time of Gaussian mixture model for each frame was $3.21 \mathrm{~s}$, while the new approach used only $0.48 \mathrm{~s}$.

The comparison results are depicted in Figure 1. It can be seen that the target pig detection results with Gaussian mixture model had misjudgment points and ghosts. Because the Gaussian mixture model did not consider the velocity changes of the moving targets, and generally the learning rate was fixed as 0.005 . When the 
learning rate (the background updating speed) and the actual scene switching speed did not match in the process of detection, the test results appear misjudgment points and ghosts. New approach could evidently enhance noise resistance, and extract the complete pigs' contours and eliminate or reduce misjudgment points and ghosts.
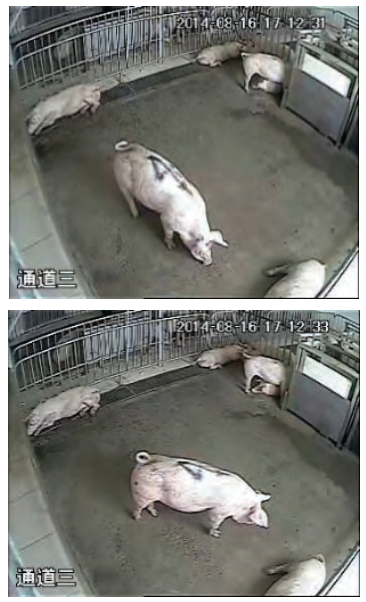

The original video frame
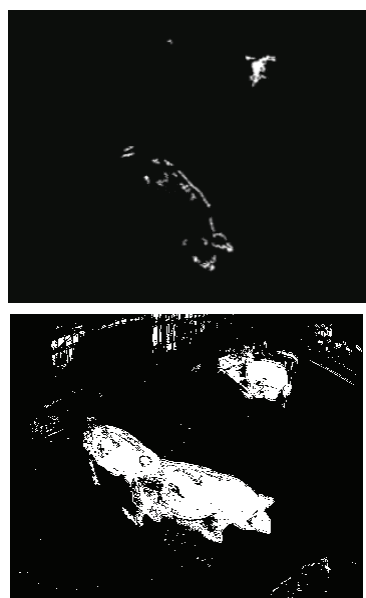

Gaussian mixture mode

Figure 1 Pig detection
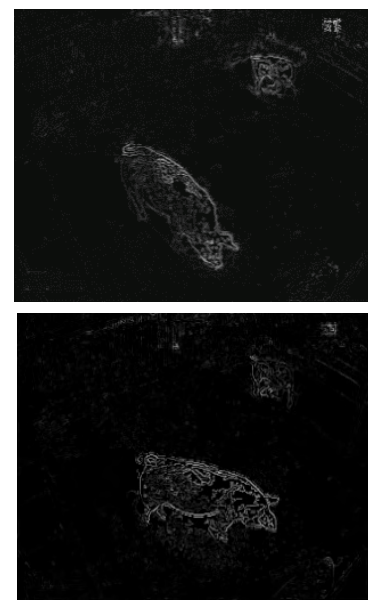

New approach

\subsection{Adaptive learning rate}

The improved algorithm used the learning rate of the adaptive selection strategy (Equation (11)) to update the background, where $T_{0}$ is the threshold of the initial video frame. When initial frame is less than $T_{0}$, then the background is being developed, a higher learning rate is needed to accelerate background modeling; When the background is developed, (the number of current video frames is larger than $T_{0}$ ) the variance is in the convergent state then the learning rate should be less than the initial learning rate of the background to maintain stability, the background updating speed and the actual scene speed is comparable to eliminate or reduce misjudgment points and ghosts.

Figure 2 is the relationship between video frames and learning rate. Based on the numerous experiments and combining the pig detection characteristics, in this paper, $T_{0}=100$ was selected, that is, set the frames before $T_{0}$ frame as the background of the initial model building stage, and use a higher degradation learning rate to speed up building the background model. The adaptive adjustments of learning rates are realized according to the actual situation of the scene. When the background model is complete after $T_{0}$ frame, the variance remained stable with a fixed learning rate $\alpha=0.005\left(T_{0}=100\right)$ in order to maintain a stable background and reduce noise interference.

Figure 3 is the detection results of different learning rate. When $T_{0}=50$, the statistical gray value is few, the initial background models can not form a stable distribution, and the following background models are difficult to achieve stable distributions; $T_{0}=150$, the following learning rate is too small to guarantee that the initial learning rate matches scene depth, misjudgment points and ghosts appeared in the image detection results. Considering the real-time feature and experimental performance, this paper chooses $T_{0}=100$.

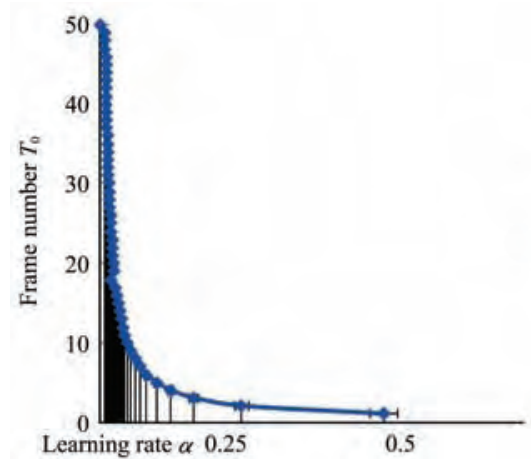

a. $T_{0}=50$

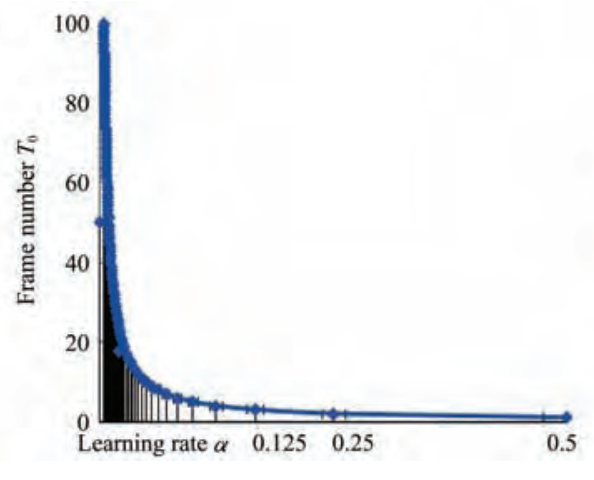

b. $T_{0}=100$

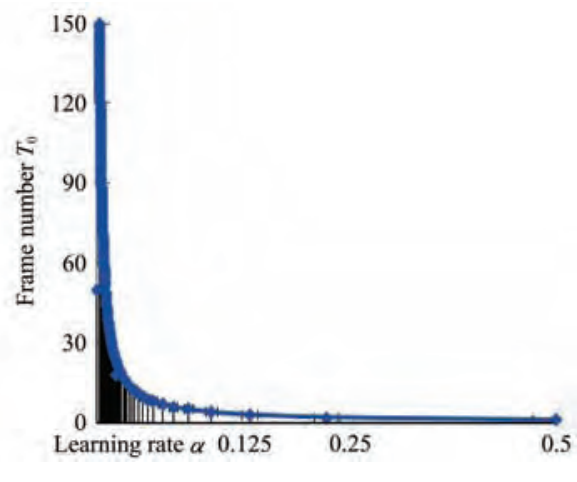

c. $T_{0}=150$

Figure 2 Relationship between video frame and learning rate 


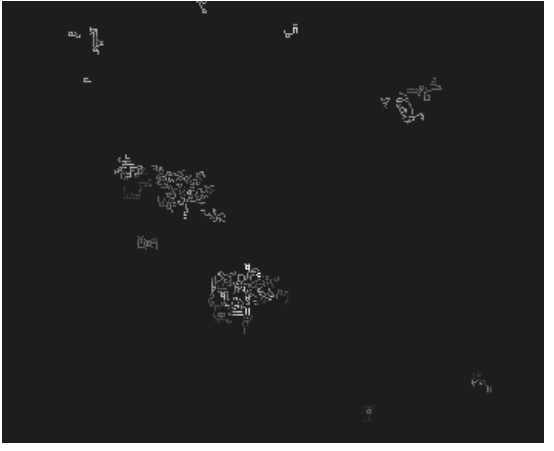

a. $T_{0}=50$

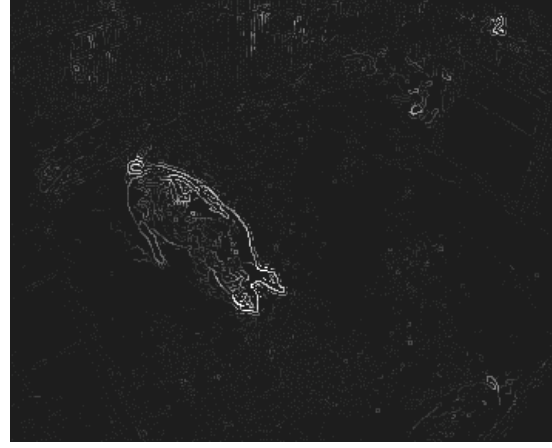

b. $T_{0}=100$

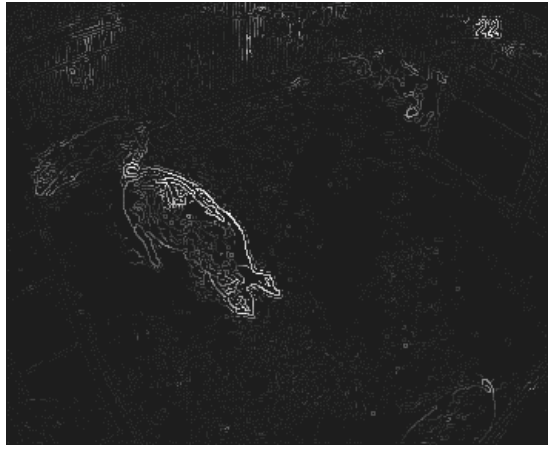

c. $T_{0}=150$

Figure 3 Detection results of different learning rate

\subsection{Delete the redundant Gaussian distributions}

The improved algorithm scans Gaussian distributions every $m$ frame to detect the weights of every Gaussian distributions. If a Gaussian distribution satisfies the Equation (10), it will be determined that the Gaussian distribution is a redundant Gaussian distribution, and it will be discarded to improve the real-time attribute of the algorithm. $m$ is set to $30,60,80,100,150$ and 200, respectively and the test results are shown below. The comparison the detection results are depicted in Figure 4.

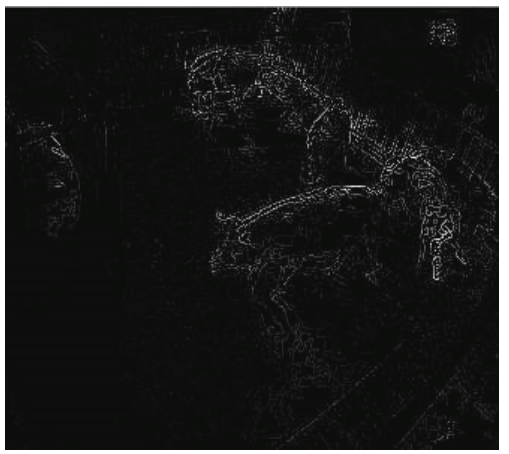

a. $m=30$

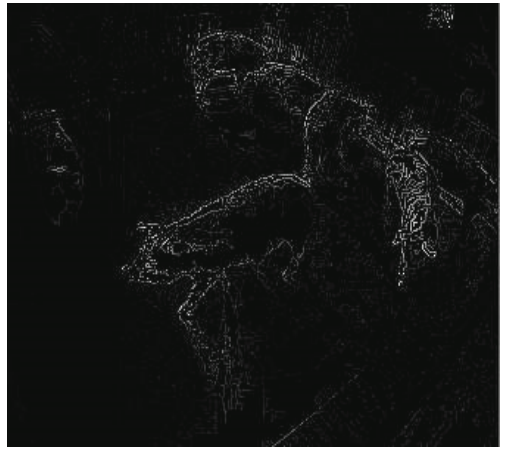

d. $m=100$

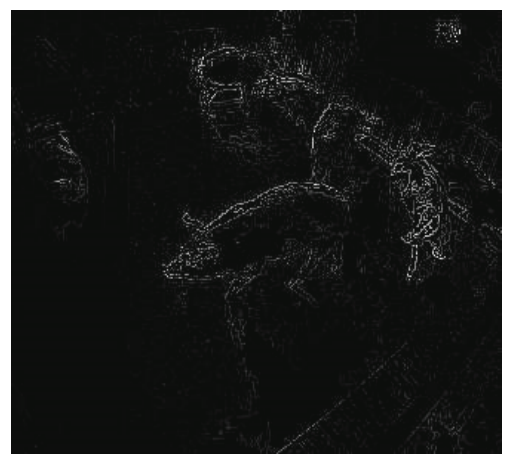

b. $m=60$



e. $m=150$

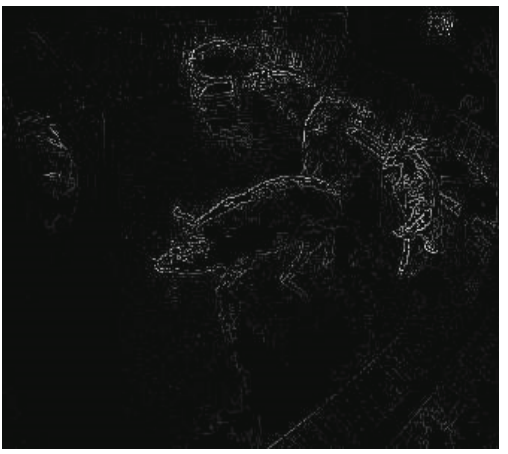

c. $m=80$

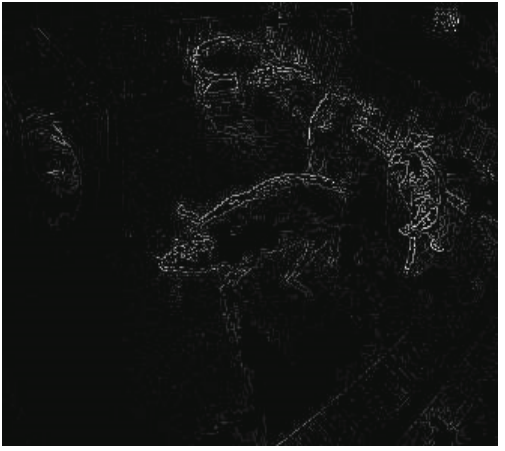

f. $m=200$

Figure 4 Detection results of different thresholds

For the threshold of frame number $m$, when the value of $m$ is small, and the running speed is slow, then the extraction is more precise and the detection is robust. If the value of the threshold $m$ is large and the running speed is fast, there will be many noise points and the detection results will be unstable. The comparison results are depicted in Figures 5 and 6.

Considering the real-time performance of algorithm and detection results, this paper chooses $m=100$.

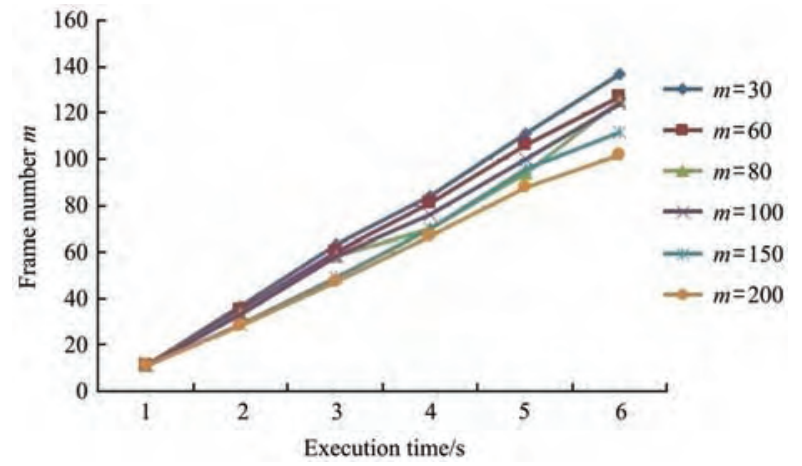

Figure 5 Comparison chart of different frame thresholds within the same time 


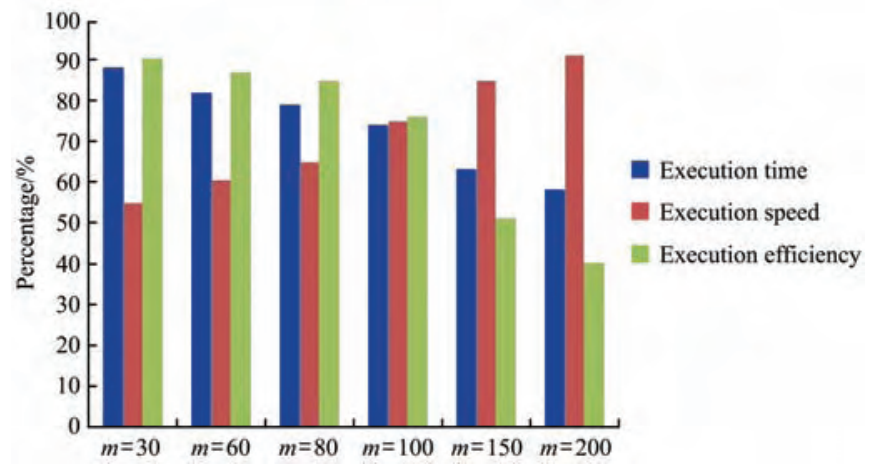

Figure 6 Comparison chart of detection performance of different frame thresholds

\section{Conclusions}

An improved self-adaptive Gaussian mixture model algorithm was proposed to overcome the drawbacks of Gaussian mixture model algorithm: in order to improve the real-time performance of algorithm, scan the Gaussian distributions with every certain number of frames, and delete the redundant Gaussian distributions, to improve the timeliness of algorithm. At the same time, different learning rates were used before and after frame number threshold to eliminate or reduce misjudgment points and ghosts, and improve the detecting accuracy of moving targets. The test results showed that the proposed method can effectively remove noise, reduce external interference, and improve the noise resistance performance.

\section{Acknowledgments}

The authors acknowledge that this research was financially supported by the National High Technology Research and Development Program of China (2013AA102306), and Independent Innovation Capability of Shandong Province (2014XGA13054).

\section{[References]}

[1] Zhu W X. Automatic identification system of pigs with suspected case based on behavior monitoring. Transactions of the CSAE, 2010; 26(1): 188-192. (in Chinese)

[2] Zhao R, Ouyang W, Li H, Wang X. Saliency detection by multi-context deep learning. IEEE Conference on Computer Vision and Pattern Recognition. IEEE, 2015; pp.1265-1274.

[3] Zhu W, Liang S, Wei Y, Sun J. Saliency optimization from robust background detection. IEEE Conference on
Computer Vision and Pattern Recognition. IEEE Computer Society, 2014; pp.2814-2821.

[4] Chang F J, Lin Y Y, Hsu K J. Multiple structured-instance learning for semantic segmentation with uncertain training data. Computer Vision and Pattern Recognition. IEEE, 2014, pp.360-367.

[5] Rubinstein M, Joulin A, Kopf J, Liu C. Unsupervised joint object discovery and segmentation in internet images. Computer Vision and Pattern Recognition. IEEE, 2013; pp.1939-1946.

[6] Zhang J, Sclaroff S, Lin Z, Shen X H, Price B, Mech R. Minimum Barrier Salient Object Detection at 80 FPS. IEEE International Conference on Computer Vision. IEEE, 2015; pp.1404-1412.

[7] Denman S, Chandran V, Sridharan S. An adaptive optical flow technique for person tracking systems. Pattern Recognition Letters, 2007; 28(10): 1232-1239.

[8] Narayana M, Hanson A, Learned-Miller E. Coherent motion segmentation in moving camera videos using optical flow orientations. IEEE International Conference on Computer Vision. IEEE, 2015; pp.1577-1584.

[9] Nguyen N, Laurendeau D, Branzan-Albu A. A robust method for camera motion estimation in movies based on optical flow. International Journal of Intelligent Systems Technologies and Applications, 2010; 9(3/4): 228-238.

[10] Huang Y, Palaniappan K, Zhuang X. Optic flow field segmentation and motion estimation using a robust genetic partitioning algorithm. IEEE Transactions on Pattern Analysis \& Machine Intelligence, 1995; 17(12): 1177-1190.

[11] Li J, Lan J. A fast moving target detection method based on the improved optical flow algorithm. Second International Conference on Electric Information and Control Engineering. IEEE Computer Society, 2012; pp.56-59.

[12] Yin J, Liu L, Li H, Liu Q. The infrared moving object detection and security detection related algorithms based on W4 and frame difference. Infrared Physics and Technology, 2016; 77: 302-315.

[13] Zhang J, Cao J, Mao B. Moving object detection based on non-parametric methods and frame difference for traceability video analysis. Procedia Computer Science, 2016; 91: 995-1000.

[14] Chen J, Zhu C, Qian Z. A moving target detection method based on integration of frame difference method and fast image segmentation. Modern Electronics Technique, 2016; 3: $13-17$

[15] Yadav D K, Singh K. A combined approach of Kullback-Leibler divergence and background subtraction for moving object detection in thermal video. Infrared Physics and Technology, 2016; 76: 21-31.

[16] Hu Y, Sirlantzis K, Howells G, Ragot N, Rodriguez P. An 
online background subtraction algorithm deployed on a NAO humanoid robot based monitoring system. Robotics and Autonomous Systems, 2016; 85(1): 37-47.

[17] Zhao K X, He D J. Target detection method for moving cows based on background subtraction. Int J Agric \& Biol Eng, 2015; 8(1): 42-49.

[18] Han B, Davis L S. Density-based multifeature background subtraction with support vector machine. IEEE Transactions on Pattern Analysis and Machine Intelligence, 2012; 34(5): 1017-1023.

[19] Porto S M C, Arcidiacono C, Anguzza U, Cascone G. A computer vision-based system for the automatic detection of lying behaviour of dairy cows in free-stall barns. Biosystems Engineering, 2013; 115(2): 184-194.

[20] Bouttefroy P L M, Bouzerdoum A, Phung S L, Beghdadi A. On the analysis of background subtraction techniques using Gaussian Mixture Models. IEEE International Conference on Acoustics, Speech and Signal Processing. IEEE, 2010; pp.4042-4045.

[21] Kayabol K, Kutluk S. Bayesian classification of hyperspectral images using spatially-varying Gaussian mixture model. Digital Signal Processing, 2016; 59: 106-114.

[22] Shah M, Deng J D, Woodford B J. Video background modeling: recent approaches, issues and our proposed techniques. Machine Vision and Applications, 2014; 25(5):
1105-1119.

[23] Jie T, Dong H, Qiuxia H, Xiaoyi M. Segmentation of wheat rust lesion image using PCA and Gaussian mix model. Transactions of the CSAM, 2014; 45(7): 267-271. (in Chinese)

[24] Liu D, Zhao K X, He D J. Real-time target detection for moving cows based on Gaussian mixture model. Transactions of the CSAM, 2016; 47(5): 288-294. (in Chinese)

[25] Kaewtrakulpong P, Bowden R. An improved adaptive background mixture model for real-time tracking with shadow detection. Video-Based Surveillance Systems. Springer US, 2002; pp.135-144.

[26] Stauffer C, Grimson W. Adaptive background mixture models for real-time tracking. Proceedings of the IEEE Computer Society Conference on Computer Vision and Pattern Recognition, 1999; 2: 246-252.

[27] Chen Z, Ellis T. A self-adaptive Gaussian mixture model. Computer Vision and Image Understanding, 2014; 122(5): 35-46.

[28] Ren K Q, Zhang P H, Xie B. Adaptive learning algorithm for moving target detection based on Gaussian mixture model. Computer Engineering and Design, 2014; 35(3): 968-974. (in Chinese)

[29] Dong X S, Chen G, Bian Z G. Improved moving object detection method based on Gaussian mixture mode. Journal of Applied Optics, 2012; 33(5): 877-883. (in Chinese) 